\title{
USO DE SIMULADORES PhET PARA EL APRENDIZAJE DEL CONCEPTO DE SOLUCIONES DESDE LAS REPRESENTACIONES EN QUÍMICA
}

\author{
USE OF PhET SIMULATORS \\ FOR LEARNING THE CONCEPT \\ OF SOLUTIONS FROM \\ REPRESENTATIONS IN CHEMISTRY
}

\section{Andis Rafael Pacheco ${ }^{1}$}

\section{Danny José Lorduy²}

\section{Elvira Patricia Flórez ${ }^{3}$}

\section{Julio César Páez ${ }^{4}$}

Grupo de Investigación en Ciencias Naturales y Educación Ambiental, GICNEA. Facultad de Educación y Ciencias Humanas. Universidad de Córdoba, Colombia.

$1 \quad$ Candidato a Magíster en Didáctica de las Ciencias Naturales, Universidad de Córdoba, Colombia. Correo: apachecoaguilar@correo.unicordoba.edu.co_ ORCID: https://orcid.org/0000-0001-9768-5875

2 Químico, Magíster en Didáctica de las Ciencias Naturales, Universidad de Córdoba, Colombia. Docente e investigador en GICNEA. Correo: dlorduyflorez@correo. unicordoba.edu.co ORCID: https://orcid.org/0000-00018355-6669

3 Doctora en Ciencias de la Educación. Coordinadora, docente e investigadora en GICNEA. Correo: epatriciaflorez@correo.unicordoba.edu.co ORCID: https:// orcid.org/0000-0003-4621-8382

$4 \quad$ Magíster en Enseñanza de las Ciencias Exactas y Naturales. Docente e investigador en GICNEA. Correo: juliopaez@correo.unicordoba.edu.co ORCID: https://orcid. org/0000-0002-6061-0316

\section{RESUMEN}

El presente artículo tiene como objetivo analizar el uso de simuladores PhET asociados con actividades experimentales para el aprendizaje del concepto de soluciones desde las representaciones en química en 40 estudiantes de grado décimo de una Institución Educativa pública del departamento de Córdoba, Colombia. La investigación se desarrolló desde una perspectiva cualitativa con alcance interpretativo, basada en la Teoría Cognitiva del Aprendizaje Multimedia (TCAM), abordando el uso de palabras e imágenes como principio para la construcción del aprendizaje, mediante la observación no participante, diarios de clase 
y guías de entrevistas en profundidad. Los resultados muestran que el uso de simuladores $\mathrm{PhET}$ asociados con actividades experimentales promovieron la construcción de aprendizajes mejor estructurados y más profundos en torno al concepto de soluciones químicas por parte de los estudiantes, mediante la movilidad mental entre la información abstracta e implícita y representaciones macro o explícitas. La investigación contribuyó significativamente a una nueva visión en las clases de química, brindando alternativas de aprendizaje de forma dinámica e interactiva con diferentes recursos que se pueden aplicar a todos los contenidos curriculares de un curso de química.

\section{PALABRAS CLAVE:}

Aprendizaje; representaciones en química; soluciones químicas; simuladores PhET; Teoría Cognitiva del Aprendizaje Multimedia.

\section{ABSTRACT}

This article aims to analyze the use of PhET simulators associated with experimental activities for learning the concept of solutions from representations in chemistry in forty tenth grade students from a public Educational Institution in the department of Córdoba, Colombia. The research was developed from a qualitative perspective with an interpretive scope, based on the Cognitive Theory of Multimedia Learning (TCAM), addressing the use of words and images as a principle for the construction of learning, through non-participant observation, class diaries and guides of in-depth interviews.The results show that the use of PhET simulators associated with experimental activities promoted the construction of better structured and deeper learning around the concept of chemical solutions by the students, through mental mobility between abstract and implicit information and macro representations or explicit. The research contributed significantly to a new vision in chemistry classes, providing learning alternatives in a dynamic and interactive way with different resources that can be applied to all the curricular contents of a chemistry course.

\section{KEYWORDS:}

Learning; representations in chemistry; chemical solutions; PhET simulators; Cognitive Theory of Multimedia Learning.

\section{INTRODUCCIÓN}

El avance de las tecnologías en los últimos años ha crecido exponencialmente, ocupando un espacio cada vez mayor en nuestra vida diaria, trayendo sus influencias en la sociedad en casi todos los campos de la acción humana y el conocimiento social. En general, ya no es posible concebir un mundo sin la tecnología actual, en donde las sociedades interactúan con nuevos conocimientos que se generan constantemente (Lorduy \& Naranjo, 2020b). Sin embargo, la educación en ciencias sigue privilegiando aspectos teóricos de forma compleja y abstracta y sin una aplicación directa en el contexto de los estudiantes (Caamaño, 2018).

Desde el punto de vista de la enseñanza de las ciencias y en particular de la asignatura de Química, en Colombia sigue tendencias propuestas por lineamientos curriculares implementados en las instituciones públicas de educación secundaria (Cárdenas \& Martínez, 2017). Esta se centra, generalmente, en la transmisión de información, ocasionando tensiones entre las metodologías planeadas por los docentes y sus acciones en el aula, de modo que se limita a la memorización, formulación y cálculos (Avila et al., 2020). Además, la enseñanza y aprendizaje de conceptos químicos, es limitado o inadecuado uso de laboratorios en el área de Ciencias Naturales, especialmente en asignaturas como química, debido a la falta de infraestructura, equipos e 
insumos que afectan los procedimientos para la ejecución de prácticas experimentales.

Por otro lado, la química participa en el desarrollo científico y tecnológico de la sociedad, por lo que, el aprendizaje de esta disciplina debería estar enfocado en comprender las transformaciones de hechos cotidianos, para dar una explicación razonable de lo que ocurre en el contexto (Caamaño, 2011, 2018). Lo anterior permite a los estudiantes indagar la información proveniente de diversas fuentes, convirtiéndose en individuos y ciudadanos capaces de tomar decisiones de forma autónoma (Meroni et al., 2015).

\section{REPRESENTACIONES EN QUÍMICA Y DIFICULTADES EN EL APRENDIZAJE DE CONCEPTOS}

La literatura especializada sobre la enseñanza de las ciencias (Gilbert \& Treagust, 2009; Taber, 2013; Talanquer, 2011), señala que las dificultades en el aprendizaje de la química son, principalmente, la existencia de diferentes representaciones durante el aprendizaje de conceptos químicos. Estas conforman el triplete químico y son etiquetadas de la siguiente forma: el macro, que hace referencia a lo que se percibe con los sentidos, tangible y explícito de un fenómeno; submicro a las entidades implícitas de las sustancias, en donde se encuentran los modelos, cargas, fotones, entre otros y lo simbólico al lenguaje verbal, escrito o expresiones matemáticas (Galagovsky et al., 2014).

En la última década, ha aumentado el interés de investigadores y docentes por aplicar software educativos como alternativa práctica y fuente de conocimientos en educación en ciencias (Cantón et al., 2017; Lorduy \& Naranjo, 2020b; Merino, 2018; Mosalve \& Monsalve, 2015; Valdez, 2017). Por tanto, pueden ser utilizados por docentes y estudiantes de forma individual, debido a que son diseñados e implementados como "puentes" entre la enseñanza y el aprendizaje de conceptos abstractos, permitiendo visualizar fenómenos físicos y químicos, promoviendo un mayor interés y comprensión del contenido presentado (Lorduy \& Naranjo, 2020a).

\section{SOFTWARE EDUCATIVO APLICADOS A LA EDUCACIÓN EN CIENCIAS}

Lossoftware educativosse pueden agruparsegún sus características y ventajas, y uno de los tipos más destacados son los simuladores (Valdez, 2017). Estos son potentes recursos didácticos para la enseñanza de diversas disciplinas, gracias a que permiten modelar fenómenos naturales, difíciles de observar en la realidad (Lorduy \& Naranjo, 2020b; Mosalve \& Monsalve, 2015). No obstante, en esta investigación, se hace énfasis sobre los simuladores PhET, los cuales son diseñados para el fortalecimiento investigativo y científico, a través de la exploración de escenarios educativos (Díaz, 2017; Valdez, 2017). De acuerdo con lo anterior, la ventaja que proveen estos simuladores en la educación en química es la interacción entre lo que perciben los estudiantes en su contexto y lo invisible o información implícita de las sustancias. Esto genera una mayor participación durante el aprendizaje, porque permite manipular las variables, "visualizarlas" y verificar los resultados, desde el punto de vista del análisis de lo que sucede con el fenómeno (Cantón et al., 2017).

En ese mismo sentido, para investigar cómo los estudiantes razonan sobre los fenómenos relacionados con los recursos utilizados, se utiliza la Teoría Cognitiva del Aprendizaje Multimedia (TCAM) (Mayer, 2019; Raviolo, 2019). Esta enfatiza que el uso de imágenes y palabras durante el proceso de enseñanza y aprendizaje pueden ser más eficientes para los estudiantes que el uso de palabras aisladas o metodologías abstractas (Díaz, 2017; Raviolo, 2019). Por lo tanto, el aprendizaje multimedia se vincula con tres procesos esenciales para el aprendizaje 
activo: la selección del material relevante, la organización del material seleccionado y la integración del material seleccionado con el conocimiento existente. La elección del material relevante está relacionada cuando el estudiante presta atención a las imágenes y palabras contenidas en los recursos presentados. La organización de los materiales seleccionados ayuda a establecer relaciones y estructuras de diferentes elementos. La integración consiste en establecer relaciones entre la información recibida y las partes relevantes del conocimiento existente. La Figura 1 representa un modelo cognitivo y los tipos de pensamientos utilizados en el aprendizaje multimedia y relaciona el sistema de procesamiento de información en los estudiantes.

Figura 1.

Modelo cognitivo sobre el aprendizaje multimedia

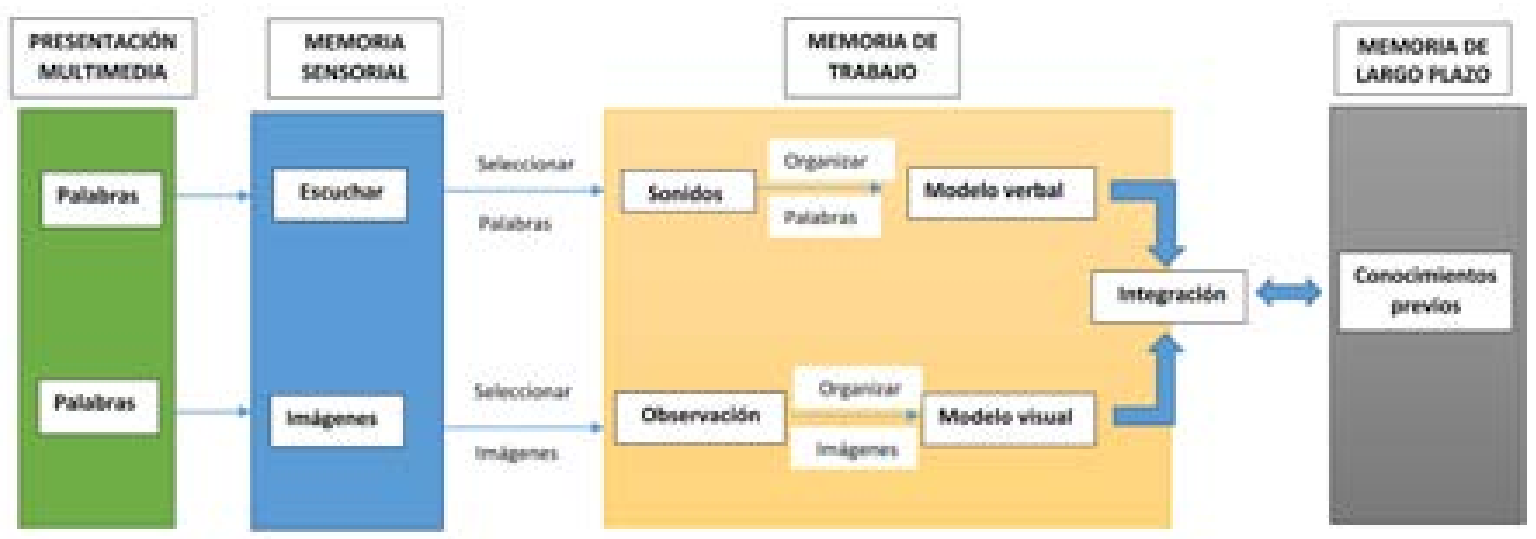

Fuente: Elaboración por los autores, según Mayer (2019).

Sin embargo, durante la enseñanza de la química, se observa que aún existe cierta resistencia por parte de los estudiantes de educación secundaria para aprender los conceptos. Esto se evidencia en los estudiantes de grado décimo de la Institución Educativa pública Francisco José de Caldas, en el departamento de Córdoba, Colombia. Dado que los docentes están restringidos al uso de pizarras, marcador y libros de texto de química, empleando conceptos abstractos, cálculos y simbologías que no trascienden a la aplicabilidad contextual de los estudiantes. Además, las prácticas experimentales en laboratorios tradicionales son un obstáculo debido a que no se vinculan recursos tecnológicos para su desarrollo y se restringen al constante uso de guías prestablecidas para desarrollarlas. Por lo que, es necesario replantear nuevas metodologías didácticas y pedagógicas que respondan a las necesidades de los estudiantes y la sociedad que los rodea, en donde se aprenda esta ciencia a través de experiencias de forma interactiva, participativa y aplicada al contexto.

Envirtud de loanterior, esta investigación defiende la premisa de que el uso de modelos simulados por software asociados a las actividades experimentales genera aprendizajes profundos, desarrollo de habilidades de pensamiento, interpretación y comprensión de conceptos químicos. Por tanto, la presente investigación gira en torno al siguiente interrogante principal, ¿Cómo el uso de simuladores PhET asociados con las prácticas experimentales promueven el aprendizaje del concepto de soluciones desde las representaciones en química en estudiantes de media académica? 


\section{METODOLOGÍA}

La investigación se desarrolló desde una perspectiva cualitativa con alcance interpretativo, estableciendo los elementos epistemológicos, conceptuales y metodológicos para aplicarse en la educación en ciencias, a través de acciones didácticas que vincularan a los estudiantes como sujetos activos del proceso (Galagovsky et al., 2014). Sin embargo, estos no se consideraron representativos del universo de estudiantes que cursaban la asignatura de química en el nivel educativo seleccionado.

El estudio se realizó teniendo en cuenta las siguientes fases: i) implementación de una guía experimental que contenía el concepto de soluciones químicas y ii) implementación de una guía simulada del mismo concepto utilizando simuladores PhET. Para ambos casos se analizaron las representaciones en química o modelos mentales de los estudiantes y su aprendizaje progresivo del concepto abordado.

\section{PARTICIPANTES Y CONTEXTO}

En este estudio participaron 40 estudiantes (E) del grado décimo de educación secundaria obligatoria de la Institución Educativa pública Francisco José de Caldas, ubicada en el departamento de Córdoba, Colombia. Para la escogencia de los estudiantes se aplicó el método de muestreo intencional o razonado, que es de tipo no probabilístico, y que por lo general implican un juicio personal o clara intención de definir o seleccionar la población con un criterio preestablecido (Cerda, 1993; Otzen \& Manterola, 2017).

\section{TÉCNICAS E INSTRUMENTOS}

Para efectos de la investigación se tomaron los instrumentos de sistematización y análisis las guías de entrevistas en profundidad y observación no participante, aplicadas a los estudiantes de grado décimo de educación secundaria. La elección de la institución siguió los criterios necesarios para la realización de la investigación, la cual debía contar con una sala de informática en funcionamiento y autorización de la dirección para realizar las actividades junto con el docente responsable del componente curricular de química en la institución. La edad, nivel socioeconómico y el género no se tomaron en cuenta en la investigación, pero es importante señalar que, la comunidad escolar involucrada en la investigación presentaba una variedad de tipos de estudiantes, personalidades, pensamientos y culturas.

\section{ANÁLISIS DE DATOS}

En el análisis de los datos recolectados en las guías de entrevista y diarios de clase de los estudiantes, se utilizó la técnica de Análisis de Contenido Cualitativo [ACC] (Krippendorff \& Bock, 2009; Lorduy \& Naranjo, 2020a). Esta se basó en los discursos de los estudiantes participantes y su contenido escrito y verbalizado. Los datos de este estudio fueron obtenidos a partir de respuesta dadas por los estudiantes a través de guías de entrevista y material impreso, durante 3 clases de aproximadamente 2 horas cada una y fueron analizados con el software Atlas ti, versión 8.4. Para lograr los objetivos propuestos en el ACC se siguieron los pasos propuestos por la técnica: I) preanálisis, II) tratamiento e interpretación de resultados y III) triangulación de la información.

PREANÁLISIS: en esta fase de organización se sistematizó las ideas iniciales con el fin de realizar un esquema preciso del desarrollo de operaciones sucesivas en un plan de análisis. Para esta etapa de la investigación se preparó el contenido del material impreso a analizar, obtenidos de la recopilación de datos en la guía de entrevista y en la observación no participante, permitiendo crear unidades hermenéuticas, en las que se siguieron normas analíticas de contenido y modelos paso a paso sin utilizar cuantificación (Lorduy \& Naranjo, 2020a; Strauss \& Corbin, 2002). 
Tratamiento de datos: Esta etapa de análisis se dio a través de la interpretación de los datos obtenidos, generando una serie de códigos que se condensaron en categorías emergentes, permitiendo concretar la información que se utilizó en los análisis, teniendo en cuenta los principios de TCAM. Finalmente, en la codificación selectiva se llevó a cabo una comparación de las categorías de la investigación con los referentes teóricos, para confrontarlas con los fragmentos de las entrevistas en profundidad.

\section{TRIANGULACIÓN DE LA INFORMACIÓN}

El proceso de triangulación permitió el cruce dialéctico con los datos obtenidos de los distintos instrumentos de recolección de información (guías de entrevista, observación no participante y diarios de clase) con el fundamento teórico descrito en esta investigación.

\section{RESULTADOS Y DISCUSIONES}

Al iniciar la investigación, se observó que los estudiantes participantes presentaban desempeños bajos, sintiéndose desmotivados durante el desarrollo de las clases de química y presentaban las actividades con el único propósito de aprobar la asignatura.

\section{FASE: ACTIVIDADES EXPERIMENTALES DIRECTAS.}

A partir de las entrevistas, observación no participante y diarios de clase de los estudiantes durante la práctica experimental sobre ácidos y bases químicas, se indagó los conocimientos previos de los estudiantes acerca de este contenido en particular. Así, se observó en la mayoría de las respuestas de los estudiantes (E1, E3, E2, E7, E8) que presentaban definiciones y representaciones con errores conceptuales de la siguiente manera:
"Las fórmulas que tienen $\mathrm{H}$ son ácidas y las que tienen $\mathrm{O}$ y $\mathrm{H}$ son bases" (E5);

"Ácido de sabor amargo, irrita los ojos y la boca" (E2);

"Bases como agua, jugo y leche" (E7).

Analizando los enunciados anteriores, el del estudiante E5 clasificó los ácidos según la presencia del elemento hidrógeno $(\mathrm{H})$ y las bases como compuestos que presentan oxígeno (O) e hidrógeno $(H)$. En este sentido, no pueden diferenciar el elemento $\mathrm{H}$ para su ion $\mathrm{H}^{+}$y la representación de hidroxilo $\mathrm{OH}^{-}$en lugar de $\mathrm{OH}$. Así, las representaciones de los estudiantes pueden relacionarse con la forma en que se enseña la asignatura de química en el aula, ya sea que estén enfocadas a la memorización, cálculos y abstracciones sin utilizar recursos que permitan comprender los conceptos que se abordan. Por lo que, es habitual que los docentes pidan a los estudiantes que imaginen situaciones abstractas en las que se están produciendo transformaciones o reacciones entre sustancias. Imaginar el fenómeno sólo desde su lenguaje verbal, generando consecuentemente dificultades en el proceso de aprendizaje de esos conceptos. En efecto, los estudiantes encuentran grandes dificultades para lograr el dominio de las ideas que involucra la utilización de distintas representaciones en el aula de clase de química (Gilbert \& Treagust, 2009). Por lo que, es necesario utilizar diferentes formas de representación de la estructura de la materia y la molecular para potenciar el proceso de aprendizaje.

En las representaciones, los estudiantes describieron el sistema de estudio en términos de sus propiedades explícitas, como el tipo de elemento o lo que percibieron con sus sentidos de forma previa. Lo anterior implica que, no infieren sobre las propiedades implícitas de las sustancias, como energías de enlaces o el 
tamaño de los átomos. En este sentido, algunos estudiantes manifiestan:

"Sí está en pequeñas cantidades no es dañino, ya que algunos alimentos, por ejemplo, las frutas, son ácidos". (E9);

"Según el ácido, en pequeñas cantidades, se pueden utilizar en alimentos como el vinagre". (E26);

"El vinagre es ácido y puedes ingerirlo sin causar ningún daño (moderadamente)". (E5).

En efecto, los estudiantes describieron las propiedades de las sustancias en términos de las representaciones macro, provistas de su memoria a largo plazo y relacionadas con sus experiencias contextuales. Además, atribuyen características a los ácidos como "malos" y a las bases como "buenas", desde aspectos alejados del conocimiento científico, lo que impide asociar sus conocimientos previos a una aplicabilidad contextual. Algunos estudiantes expresaron:

"No todos los ácidos son malos para la salud, por ejemplo, el ácido ascórbico (vitamina C) que se utiliza para combatir la gripe" (E23);

"El ácido como algo malo y la base como algo bueno" (E1);

"El ácido es algo malo para el estómago" (E8).

Por lo tanto, el conocimiento químico solo se construye cuando los estudiantes son capaces de explicar el fenómeno movilizándose a través de los vértices del triplete químico, generando inferencias a partir de diferentes escalas (Johnstone, 1991; Lorduy \& Naranjo, 2020a; Taber, 2013; Talanquer, 2011). Sin embargo, una de las dificultades en el proceso de aprendizaje de conceptos químicos es que, generalmente, se basa en solo una representación (macro) por lo que es poco probable relacionarlo con aspectos presentes a nivel submicro (Johnstone, 1982; Talanquer, 2018).
En efecto, los estudiantes no fueron capaces de dar explicaciones del fenómeno desde la escala submicro, porque no comprendían las abstracciones existentes en estas representaciones implícitas. Por tanto, es necesario que en el proceso de aprendizaje se produzca la integración de las distintas representaciones químicas de forma sistémica, más que contenidos escalonados y desconectados. En este sentido, las simulaciones son un recurso de modelización a fin de entender sus propiedades desde el triplete químico y permitir evaluar las distintas estrategias operativas o procedimentales del sistema de estudio. Ante la situación planteada, las actividades experimentales asociadas solo a lo "real o directo" no promueven aprendizajes profundos, por lo que se requieren combinarse sinérgicamente con actividades simuladas para ser incorporadas al proceso de enseñanza de las ciencias experimentales.

\section{FASE: ACTIVIDADES SIMULADAS.}

Posteriormente a la práctica experimental en el laboratorio de química, el docente utilizó los simuladores PhET, a fin de ver virtualmente, cómo se comportan los átomos y las moléculas en determinadas condiciones. Los resultados obtenidos en este punto permitieron reducir las dificultades que presentaban los estudiantes en la experiencia. En este sentido, para organizar la actividad multimedia fue necesario que el docente analizara y reflexionara sobre la cantidad de información a presentar a los estudiantes, teniendo en cuenta si era progresiva representacionalmente para promover su aprendizaje del concepto de soluciones químicas. Por tanto, se mantuvo los cinco procesos cognitivos establecidos en la TCAM (Mayer, 2019; Raviolo, 2019). Las flechas indicadas (seleccionar imágenes y organizar palabras) en la figura 1 , en el campo de la memoria de trabajo, representaron los cinco procesos lógicos que fueron necesarios para el 
aprendizaje multimedia, estos son: selección de palabras, selección de imágenes, organización de palabras e imágenes, integración. Estos procesos se muestran en la Tabla 1.

Tabla 1.

Cinco procesos cognitivos de la TCAM

\begin{tabular}{ll}
\hline \multicolumn{1}{c}{ Proceso } & \multicolumn{1}{c}{ Descripción } \\
\hline $\begin{array}{l}\text { Selección de } \\
\text { palabras }\end{array}$ & $\begin{array}{l}\text { El estudiante presta atención a las palabras más relevantes en un mensaje } \\
\text { multimedia para crear sonidos en la memoria de trabajo. }\end{array}$ \\
\hline $\begin{array}{l}\text { Selección de } \\
\text { imágenes }\end{array}$ & $\begin{array}{l}\text { El estudiante presta atención a las imágenes relevantes en un mensaje } \\
\text { multimedia para crear imágenes en la memoria de trabajo. }\end{array}$ \\
\hline $\begin{array}{l}\text { Organización de } \\
\text { palabras }\end{array}$ & El estudiante establece vínculos entre las palabras seleccionadas para \\
\hline Organización de & crear modelos verbales coherentes en la memoria de trabajo. \\
imágenes & El estudiante establece vínculos entre las imágenes seleccionadas para \\
\hline Integración & crear modelos gráficos coherentes en la memoria de trabajo. \\
& El estudiante establece vínculos entre los modelos verbales- gráficos y el \\
& conocimiento existente.
\end{tabular}

Fuente: Adaptado de Mayer (2019).

Así, se organizaron los mensajes multimedia de acuerdo con sus conocimientos previos provistos por el imaginario de los estudiantes en las prácticas experimentales, permitiendo más probabilidades de lograr un aprendizaje profundo. En ese sentido, el docente comenzó la práctica presentando el comportamiento ácido o base de un líquido, relacionando el color del líquido con el $\mathrm{pH}$, relacionando las concentraciones de sus respectivos iones. La figura 2 muestra las distintas representaciones que se analizaron en el simulador PhET, teniendo en cuenta cada uno de los procesos cognitivos de la TCAM.

Figura 2.

Simulador PhET desde las representaciones macro, submicro y simbólicas.
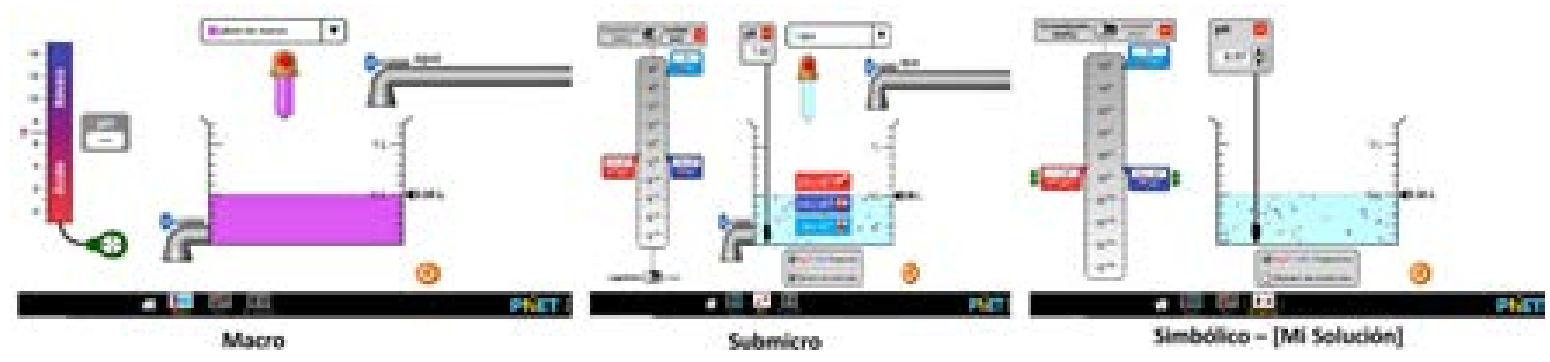

Fuente: Simulador PhET Escala de $\mathrm{pH}$.

Al analizar las representaciones se observó que los estudiantes se cuestionaban el comportamiento de las sustancias teniendo en cuenta los modelos moleculares y la función de sus cargas. Algunos estudiantes manifestaron:

"El vómito es una sustancia ácida al agregar agua, su $\mathrm{pH}$, que era 2.0, pasa a 2.8, y su 
concentración de $\mathrm{H}^{+}$disminuye y la de $\mathrm{OH}^{-}$ aumenta" (E14).

"La sangre, en cambio, al ser una de las sustancias seleccionadas más básicas, al tener un $\mathrm{pH}$ de 7,4 al añadir agua es de 7,3, su $\mathrm{H}^{+}$ aumenta y su $\mathrm{OH}$ disminuye" (E27).

A partir de los discursos de los estudiantes E14 y E27, se observó que, los estudiantes son capaces de relacionar el cambio de $\mathrm{pH}$ con la concentración de los respectivos iones $\mathrm{H}^{+}$ y $\mathrm{OH}^{-}$. En este sentido, las representaciones de las sustancias en química, que a menudo son abstractas, a través de recursos gráficos disponibles en computadoras permitieron una nueva forma de construir conocimiento en la experiencia simulada.

El principio de la TCAM utilizada para analizar este paso fue la modalidad que establece que los estudiantes aprenden más cuando utilizan la animación y la narración en lugar de la animación $y$ el texto escrito, porque en ese momento la audición y la visión se utilizan en ambos canales, lo que conduce a una comprensión más significativa $y$, en consecuencia, al aprendizaje (Mayer, 2019). En consecuencia, la información en la memoria de trabajo de los estudiantes (conocimientos previos), pudo esquematizarse a través de textos, imágenes y sonidos. Posteriormente, el conocimiento se elaboró en la memoria de trabajo, que fueron los modelos verbales e imágenes animadas descritos en la TCAM (Mayer, 2019). Por tanto, la actividad simulada permitió que los estudiantes representar la concentración de iones hidronio en la solución y explicar a partir de esto algunas de sus propiedades explícitas. En virtud de lo anterior, algunos unos estudiantes expresaron:

"Las sustancias ácidas contienen hidrógeno en su composición molecular, tienen un $\mathrm{pH}$ por debajo de 7 y tienen poder corrosivo, mientras que las sustancias básicas tienen un $\mathrm{pH}$ superior a 7 y no tienen hidrógeno en su fórmula química".
"El pH sirve para saber si las sustancias son ácidas o básicas. Las sustancias ácidas tienen una mayor cantidad de $\mathrm{H}^{+}$, mientras que las sustancias básicas tienen una mayor cantidad de $\mathrm{OH}^{-\prime}$ (E12).

Sobre lo anterior, se infiere que los estudiantes se movilizaron mentalmente hacia las representaciones abstractas e implícitas de las sustancias estudiadas (cargas eléctricas, composición molecular) y brindaron información de sus propiedades (poder corrosivo) cuando se refieren a las sustancias ácidas y básicas. En tal sentido, la interacción de los estudiantes con el simulador se basó en el principio de segmentación de la TCAM, el cual promovió el aprendizaje cuándo la actividad multimedia se presentó en pasos o segmentos adecuados y no como una unidad continua (Giordan \& Gois, 2009). Por esta razón, fueron capaces de transitar entre las distintas representaciones de una manera más organizada y estructurada, generando una movilidad mental que se esquematiza a partir del modelo cognitivo sobre el aprendizaje multimedia (Figura 3). No obstante, cuando imaginaban el fenómeno, solo desde su lenguaje o el del docente, se generó consecuentemente dificultades en el proceso de aprendizaje, ya que no lo relacionaban con el fenómeno que ocurría a nivel submicro, lo que les impedía explicarlo desde las interacciones con las representaciones macro y simbólicas. 
Figura 3.

Modelo cognitivo sobre el aprendizaje multimedia, representaciones en química y movilidad mental de los estudiantes.

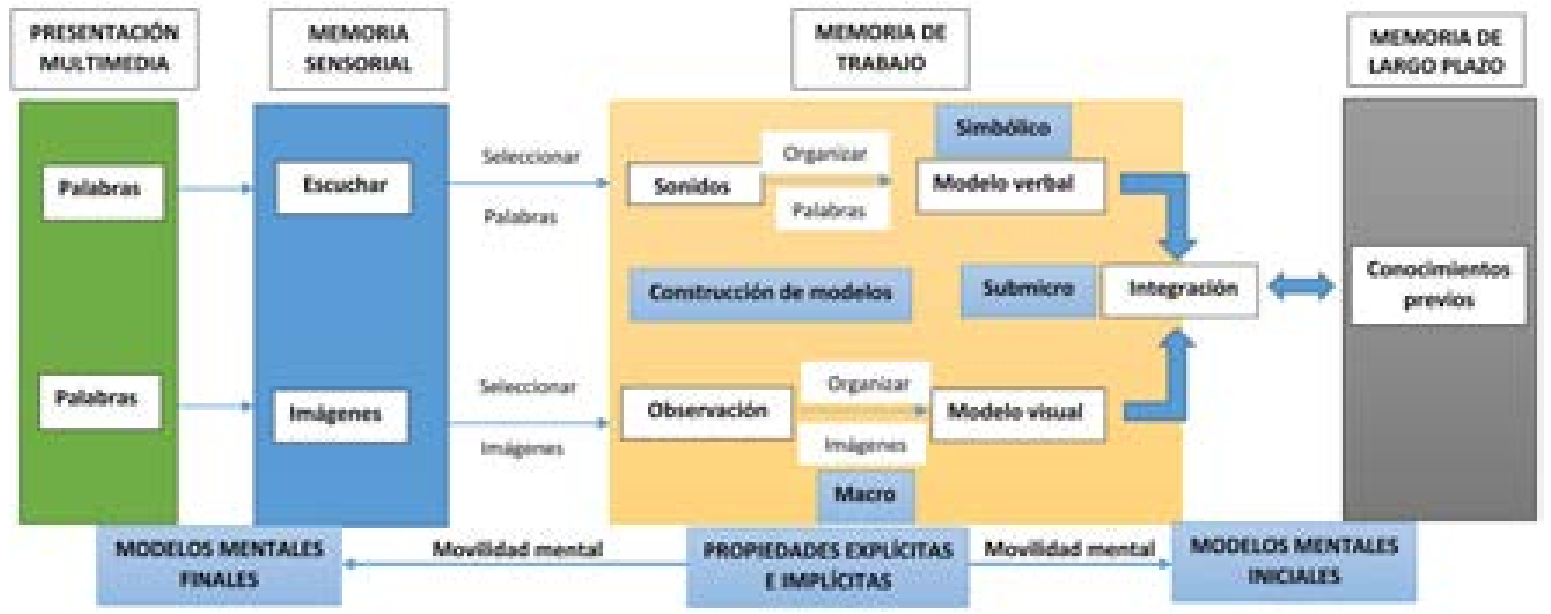

Fuente: Elaboración de los autores adaptado de Mayer (2019).

Finalmente, tanto la actividad experimental como simulada se presentaron en un momento de integración entre la teoría y la práctica, basada en discusiones, análisis e interpretación de lo observado, buscando un desarrollo conceptual y cognitivo en los estudiantes, que les permitiera resaltar los fenómenos y reconstruir sus ideas y modelos mentales iniciales a unos más estructurados. Lo anterior, se logró teniendo en cuenta los principios de la TCAM el cual demostró ser importante desde la construcción, ejecución y evaluación de las actividades realizadas para promover el aprendizaje del concepto de soluciones químicas.

\section{CONCLUSIONES E IMPLICACIONES DIDÁCTICAS}

La investigación contribuyó significativamente a una nueva visión en las clases de química, brindando alternativas de aprendizaje de forma dinámica e interactiva con diferentes recursos que se pueden aplicar a todos los contenidos curriculares. Además, proporcionó a los estudiantes nuevas formas de interactuar con sustancias químicas que no pueden hacerlo de forma directa debido a su peligrosidad biológica (vómito, sangre, saliva, ácido de batería, entre otros). Sin embargo, es importante resaltar que, el uso de simuladores no garantiza que los estudiantes aprendan, por lo que es necesario delinear objetivos y estrategias que permitan esta integración para ser eficientes en el proceso de aprendizaje.

Esta investigación permite dar cabida a la creación de secuencias didácticas (SD) que permita visibilizar la evolución del aprendizaje de los estudiantes a partir de sus modelos mentales progresivos. Este último, se considera un aporte de este estudio, pues destaca que el uso de simuladores asociados a las actividades experimentales puede ser utilizados en las clases de química como una herramienta didáctica para ayudar a docentes y estudiantes en el proceso de enseñanza y aprendizaje. En este sentido, hay que tener en cuenta los principios de la TCAM el cual demostró ser importante en la construcción, ejecución y evaluación de las actividades realizadas.

Sin embargo, no es suficiente depender de las actividades experimentales para el aprendizaje 
de conceptos químicos. En este sentido, fue necesario combinar la práctica y la modelización de fenómenos a partir de simuladores para adentrarse al mundo particulado, permitiendo la interpretación e inferencia de las propiedades de algunas sustancias en soluciones ácidas y básicas por parte de los estudiantes.

En cuanto a los simuladores PhET, como recursos multimedia, permitieron a los estudiantes modelar las representaciones macro, simbólica y submicro del concepto de soluciones químicas. Además, se evidenció que la mayoría de los estudiantes durante el aprendizaje del concepto químico abordado ya tienen conocimientos previos que les permitieron generar inferencias sobre las propiedades implícitas de las sustancias. Estas pueden tener orígenes en el entorno social, en la experiencia de vida o en las ideas definidas por la escuela. Por lo que, fueron utilizados por el docente en el aula para promover el aprendizaje más estructurado y dar sentido a la introducción de las nuevas representaciones (simbólico y submicro). Tales ideas también se corroboraron con la TCAM, que son consideradas esenciales en la construcción y reconstrucción de nuevos aprendizajes.

Finalmente, se recomienda que durante el proceso de enseñanza y aprendizaje de conceptos químicos se utilice esta innovadora herramienta tecnológica dentro de los cursos de química de educación secundaria, puesto que, permiten a los estudiantes mediar y transformar su aprendizaje en habilidades para la vida, como futuros profesionales responsables de la formación moral, intelectual y científica de la sociedad.

\section{REFERENCIAS BIBLIOGRÁFICAS}

Avila, O. D., Lorduy, D. J., Aycardi, M. P., \& Flórez, E. P. (2020). Concepciones de docentes de química sobre formación por competencias científicas en educación secundaria. Revista ESPACIOS, 41, 244260. https://doi.org/10.48082/espaciosa20v41n46p21

Caamaño, A. (2011). Enseñar química mediante la contextualización, la indagación y la modelización. Alambique, Didáctica de Las Ciencias Experimentales, 69, 21-34.

Caamaño, A. (2018). Enseñar química en contexto: un recorrido por los proyectos de química en contexto desde la década de los 80 hasta la actualidad. Educación Química, 29(1), 21. https://doi. org/10.22201/fq.18708404e.2018.1.63686

Cantón, D., Arellano, J. J., Hernández, M. Á., \& Nieva, O. S. (2017). Didactic use of immersive virtual reality with $\mathrm{NUI}$ focused on the inspection of wind turbines. Apertura, 9(2), 8-23. https://doi.org/10.32870/ ap.v9n2.1049

Cárdenas Navas, A. M., \& Martínez Rivera, C. A. (2017). Los referentes curriculares instituidos para la elaboración del conocimiento escolar en ciencias en Colombia : ¿qué caracteriza la estructura de los estándares básicos de competencias en ciencias? Enseñanza de Las Ciencias: Revista de Investigación y Experiencias Didácticas, Extra, 1183. 
Cerda, H. (1993). Los elementos de la investigación como reconocerlos, diseñarlos y construirlos. Editorial El Buho, 439. http://www.sidalc.net/cgi-bin/wxis.exe/?IsisScript=COLEC. xis\&method=post\&formato $=2 \&$ cantidad $=1$ \&expresion $=m f n=010116$

Díaz Pinzón, J. E. (2017). Importancia de la simulación Phet en la enseñanza y aprendizaje de fracciones equivalentes. Revista Educación y Desarrollo Social, 11(1), 48-63. https://doi.org/10.18359/ reds. 2011

Galagovsky, L. R., Bekerman, D., Giacomo, M. A., \& Di Alí, S. (2014). Algunas reflexiones sobre la distancia entre "hablar química" y "comprender química." Ciência \& Educação (Bauru), 20(4), 785-799. https://doi.org/10.1590/1516-73132014000400002

Gilbert, J. K., \& Treagust, D. F. (2009). Towards a Coherent Model for Macro, Submicro and Symbolic Representations in Chemical Education (pp. 333-350). https://doi.org/10.1007/978-1-4020-88728_15

Giordan, M., \& Gois, J. (2009). Entornos virtuales de aprendizaje en química: una revisión de la literatura. Educación Química, 20(3), 301-313. https://doi.org/10.1016/s0187-893x(18)30030-2

Johnstone, A. H. (1982). Macro-and micro-chemistry. In School Science Review, 64 (227), (pp. 377379).

Johnstone, A. H. (1991). Why is science difficult to learn? Things are seldom what they seem. Journal of Computer Assisted Learning, 7(2), 75-83. https://doi.org/10.1111/j.1365-2729.1991.tb00230.x

Krippendorff, K., \& Bock, M. A. (2009). The Content Analysis Reader. Sage Publications.

Lorduy, D. J., \& Naranjo, C. P. (2020a). Percepciones de maestros y estudiantes sobre el uso del triplete químico en los procesos de enseñanza-aprendizaje. Revista Científica, 39(3), 324-340. https:// doi.org/10.14483/23448350.16427

Lorduy, D. J., \& Naranjo, C. P. (2020b). Tecnologías de la información y la comunicación aplicadas a la educación en ciencias. Praxis \& Saber, 11(27), e11177. https://doi.org/10.19053/22160159.v11. n27.2020.11177

Mayer, R. E. (2019). How multimedia can improve learning and instruction. In The Cambridge Handbook of Cognition and Education (pp. 460-479). https://doi.org/10.1017/9781108235631.019

Merino, C. (2018). Enseñar ciencias para aprender a imaginar, representar y evaluar. REINECC Editorial, 2(1), 1-3. https://doi.org/10.5027/reinnec.V2.I1.31

Meroni, G., Copello, M. I., \& Paredes, J. (2015). Enseñar química en contexto. Una dimensión de la innovación didáctica en educación secundaria. Educacion Quimica, 26(4), 275-280. https://doi. org/10.1016/j.eq.2015.07.002

Mosalve, N. Y., \& Monsalve, C. (2015). La inclusión de la computadora en el aula por docentes de quinto grado de básica primaria como herramienta para propiciar el aprendizaje significativo en los estudiantes. Revista EAN, 79, 50. https://doi.org/10.21158/01208160.n79.2015.1267

Otzen, T., \& Manterola, C. (2017). Técnicas de Muestreo sobre una Población a Estudio. International Journal of Morphology, 35(1), 227-232. https://doi.org/10.4067/S0717-95022017000100037

Raviolo, A. (2019). Imágenes y enseñanza de la Química. Aportes de la Teoría cognitiva del aprendizaje multimedia. Educación Química, 30(2), 114. https://doi.org/10.22201/fq.18708404e.2019.2.67174

Strauss, A., \& Corbin, J. (2002). Bases de la investigación cualitativa: técnicas y procedimientos para desarrollar la teoría fundamentada. Universidad de Antioquia, Medellín, Colombia.

Taber, K. S. (2013). Revisiting the chemistry triplet: Drawing upon the nature of chemical knowledge and the psychology of learning to inform chemistry education. In Chemistry Education Research and Practice (Vol. 14, Issue 2, pp. 156-168). Royal Society of Chemistry. https://doi.org/10.1039/ c3rp00012e 
Talanquer, V. (2011). Macro, submicro, and symbolic: The many faces of the chemistry "triplet." International Journal of Science Education, 33(2), 179-195. https://doi. org/10.1080/09500690903386435

Talanquer, V. (2018). Chemical rationales: another triplet for chemical thinking. International Journal of Science Education, 40(15), 1874-1890. https://doi.org/10.1080 /09500693.2018.1513671

Valdez, D. (2017). Uso didáctico de Phet Simulaciones Interactivas, para la comprensión de los estados de la materia en la ciencia físico-química. http://ria.utn. edu.ar/handle/123456789/1836 